\title{
छூ
}

\section{First Observation of Two-Electron One-Photon Transitions in Single-Photon $K$-Shell Double Ionization}

\author{
J. Hoszowska, ${ }^{1, *}$ J.-Cl. Dousse, ${ }^{1}$ J. Szlachetko, ${ }^{2,3, \dagger}$ Y. Kayser, ${ }^{1}$ W. Cao, ${ }^{1, \ddagger}$ P. Jagodziński, ${ }^{3}$ \\ M. Kavčič ${ }^{4}$ and S. H. Nowak ${ }^{1}$ \\ ${ }^{1}$ Department of Physics, University of Fribourg, CH-1700 Fribourg, Switzerland \\ ${ }^{2}$ European Synchrotron Radiation Facility (ESRF), F-38043, Grenoble, France \\ ${ }^{3}$ Institute of Physics, Jan Kochanowski University, 25-406 Kielce, Poland \\ ${ }^{4}$ J. Stefan Institute, SI-1001, Ljubljana, Slovenia \\ (Received 19 April 2011; published 27 July 2011)
}

\begin{abstract}
Experimental evidence for the correlated two-electron one-photon transitions $\left(1 s^{-2} \rightarrow 2 s^{-1} 2 p^{-1}\right)$ following single-photon $K$-shell double ionization is reported. The double $K$-shell vacancy states in solid $\mathrm{Mg}, \mathrm{Al}$, and $\mathrm{Si}$ were produced by means of monochromatized synchrotron radiation, and the two-electron one-photon radiative transitions were observed by using a wavelength dispersive spectrometer. The two-electron one-photon transition energies and the branching ratios of the radiative one-electron to twoelectron transitions were determined and compared to available perturbation theory predictions and configuration interaction calculations.
\end{abstract}

Understanding electron-electron interactions in ionization, excitation, and relaxation of many-body systems is one of the key issues of atomic physics. In this context of special interest are hollow atoms, i.e., atoms with empty innermost shells and occupied outer shells, because singlephoton double $K$-shell ionization is driven by multielectron interactions (see [1,2], and references therein), and the decay of $K$-shell hollow atoms involves electron correlation effects. Furthermore, hollow atom formation in ultraintense hard $\mathrm{x}$-ray free-electron laser beams reveals electron dynamics on the femtosecond time scale [3].

In response to a $K$-shell doubly excited state, electron relaxation and rearrangement processes follow. The excited atom decays in a cascade of nonradiative Auger and radiative transitions. The radiative decay of double $K$-shell hole states proceeds mainly through the oneelectron one-photon (OEOP) process, which corresponds to the $K \alpha^{h}\left(1 s^{-2} \rightarrow 1 s^{-1} 2 p^{-1}\right)$ hypersatellite transition. In the few orders of magnitude weaker competitive decay channel, the two-electron one-photon (TEOP) transition $K \alpha \alpha^{h}\left(1 s^{-2} \rightarrow 2 s^{-1} 2 p^{-1}\right)$, the two $K$-shell core holes are filled simultaneously via a correlated two-electron jump and one photon is emitted (see Fig. 1). TEOP transitions are thus correlated multielectron processes which can be described only by many-electron models.

Interest in TEOP transitions dates back to 1925. Predicted by Heisenberg [4], it was only 50 years later that the first experimental evidence for TEOP transitions in heavy-ion (HI) collisions was reported by Wölfli et al. [5]. Although the $K \alpha^{h}$ to $K \alpha \alpha^{h}$ branching ratio should not depend on the excitation mode, multiple electron ionization in $\mathrm{HI}$ collisions changes the electronic configurations and affects the intensities and energies of the measured transitions. Thus data from HI collision experiments show a wide spread of values [6-10], making comparison with theory often inconclusive. On the theoretical side, significant differences in the predicted TEOP radiative decay rates have been reported [11-21]. In this respect, photon impact data provide a more stringent test for atomic structure calculations. However, the single-photon double $K$-shell ionization cross sections are $\sim 10^{3}$ smaller than in HI collisions. Thus photoionization experiments are more challenging, and, to the best of our knowledge, all attempts to measure the TEOP transitions have been unsuccessful so far (see, e.g., [22]).

In this Letter, we present the first observation of the correlated two-electron one-photon transitions of $\mathrm{Mg}, \mathrm{Al}$, and $\mathrm{Si}$ in single-photon $K$-shell double ionization. Our objective was to determine the branching ratios and the TEOP transition energies and to demonstrate the potential of the TEOP radiative decay of $K$-shell hollow atoms to unravel inter- and intrashell electron correlations in manyelectron systems.

The experiments were carried out at the $\mathrm{x}$-ray microscopy beam line ID21 of the European Synchrotron Radiation Facility. The beam produced by two undulators

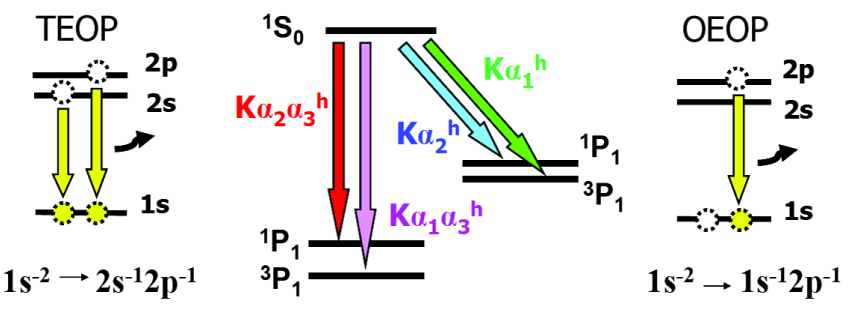

FIG. 1 (color online). Schematic of the TEOP (left) and OEOP (right) transitions and the atomic level decay diagram for the initial $K$-shell two-hole state ${ }^{1} S_{0}$ (middle). 

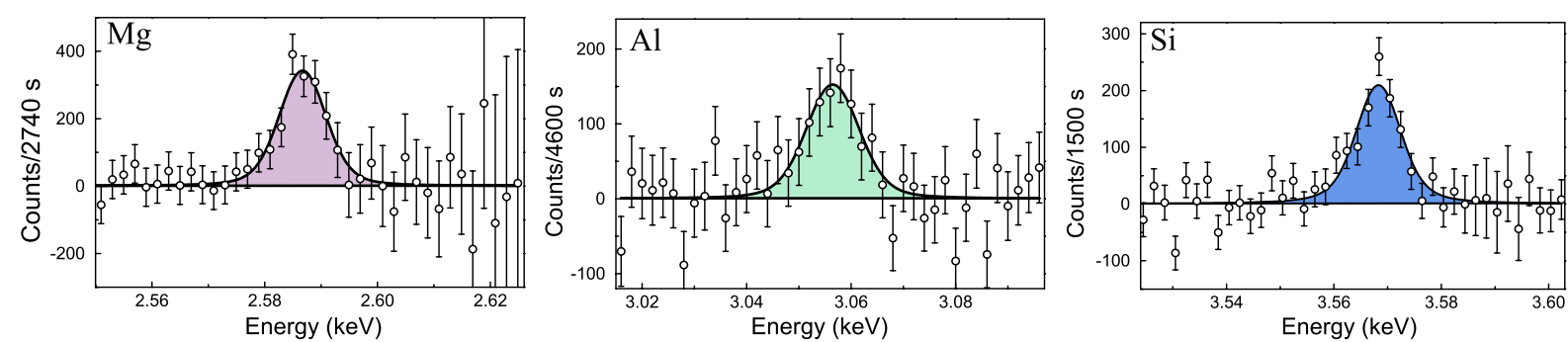

FIG. 2 (color online). Two-electron one-photon transitions of $\mathrm{Mg}, \mathrm{Al}$, and $\mathrm{Si}$. As for the studied elements the $L-S$ coupling scheme prevails, the ${ }^{3} P_{1} \rightarrow{ }^{1} S_{0}$ is forbidden by the $E 1$ selection rules, and only the ${ }^{1} P_{1} \rightarrow{ }^{1} S_{0}$ transition is observed. The solid lines correspond to the best least-squares fits to the data using Voigt functions.

was monochromatized by using the double $\mathrm{Ni} / \mathrm{B}_{4} \mathrm{C}$ multilayer monochromator, and the upper harmonics were rejected with Ni-coated mirrors. Photon energies in the region of the $K$-shell double photoionization crosssection maxima [2] of $3.364 \mathrm{keV}$ for $\mathrm{Mg}$ and $4.620 \mathrm{keV}$ for $\mathrm{Al}$ and $\mathrm{Si}$, respectively, were chosen. The microfocused incident photon flux was $\sim 2-3.5 \times 10^{12}$ photons/s.

Measurements of the x-ray spectra were performed by means of the ID21 wavelength dispersive spectrometer (WDS) [23]. The high efficiency and good energy resolution of the WDS were prerequisites for the experiment. The WDS is based on a polycapillary optics for x-ray fluorescence collection, a flat crystal, and a flow gas x-ray detector. The spectrometer was equipped with a $\mathrm{Si}(111)$ crystal for the $\mathrm{Mg}$ and $\mathrm{Al}$ measurements and a $\mathrm{Ge}(220)$ crystal for the Si ones. Self-supported metallic foils of $\mathrm{Mg}, \mathrm{Al}$, and a $c$-Si were employed. The $\mathrm{Al}$ and $\mathrm{Si}$ sample purity was 99.999\% and that of $\mathrm{Mg} 99.9 \%$. The energy calibration of the WDS was based on the recommended energies [24] assigned to the measured $\mathrm{Rh}, \mathrm{Ru}, \mathrm{Cl}, \mathrm{Pd}, \mathrm{Ag}, \mathrm{Sn}, \mathrm{K}$, and $\mathrm{Sc}$ diagram transitions. These also served to determine the full width at half maximum (FWHM) of the Gaussian instrumental response function. The FWHM was found to be in the 7-10 eV range. The $\mathrm{x}$-ray spectra were collected in successive scans of $\sim 0.5-1 \mathrm{~h}$ each, with total acquisition times of $\sim 42, \sim 51$, and $\sim 17 \mathrm{~h}$, for $\mathrm{Mg}, \mathrm{Al}$, and $\mathrm{Si}$, respectively. For normalization purposes the photon flux was recorded at the beginning and the end of each scan.
The TEOP transitions of $\mathrm{Mg}, \mathrm{Al}$, and $\mathrm{Si}$ are shown in Fig. 2. To derive the $K \alpha \alpha^{h}$ residual spectra from the measured data sets, the background and the $K$ x-ray spectra of trace elements in the case of $\mathrm{Mg}$ and $\mathrm{Si}$ (see Fig. 3) were subtracted beforehand. The $\mathrm{Cl} K \alpha$ and $\mathrm{K}$ $K \beta$ spectral profiles were determined from complementary measurements using the WDS and also the von Hamos high-resolution x-ray spectrometer of Fribourg [25]. For illustration, the high-resolution spectra are depicted in the insets in Fig. 3.

To determine the branching ratios (BR), we took advantage of the well known values of the single [26] and the double [2] $K$-shell photoionization cross sections and, thus, adopted an approach based on the intensities of the closelying reference $K$ x-ray diagram transitions instead of those of the hypersatellites. In fact, the latter could not be measured with the same crystal, and in addition corrections for the polycapillary transmission would have been important. The following expression was employed:

$$
\mathrm{BR}=\frac{I^{r}}{I^{K \alpha \alpha^{h}}} \frac{\sigma_{K K}}{\sigma_{K}^{r}} \frac{n}{n^{r}} \frac{\omega_{K K}}{\omega_{K}^{r}} F_{\text {expt }},
$$

where $I^{K \alpha \alpha^{h}}$ and $I^{r}$ stand for the intensities of the $K \alpha \alpha^{h}$ and the reference $K$ x-ray transitions, respectively. $\sigma_{K K}$ and $\sigma_{K}^{r}$ are the double and single $K$-shell photoionization cross sections, $\omega_{K K}$ and $\omega_{K}^{r}$ are the fluorescence yields for the double- and single-hole states, and $n$ and $n^{r}$ denote the number of atoms per unit volume. $F_{\text {expt }}$ corresponds to the
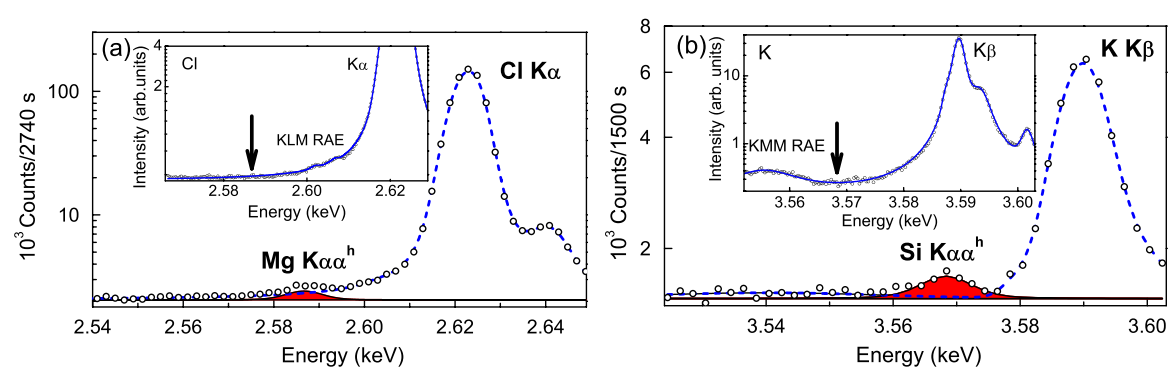

FIG. 3 (color online). X-ray spectra of $\mathrm{Mg}$ (a) and $\mathrm{Si}$ (b) showing the TEOP transitions (filled areas) and the $\mathrm{Cl} K \alpha$ and $\mathrm{K} K \beta$ transitions due to trace impurities (dashed lines). Insets represent the high-resolution $\mathrm{x}$-ray spectra of $\mathrm{Cl}$ (a) and $\mathrm{K}$ (b) comprising the diagram lines and the accompanying $K L M$ or $K M M$ radiative Auger effect (RAE) transitions [30]. As shown, the $K \alpha \alpha^{h}$ energy regions (indicated by arrows) are free of additional spectral features. 
experimental correction factor accounting for the relative differences in the photon flux, crystal reflectivity, detector efficiency, polycapillary transmission, self-absorption, and relative transition probabilities of the $K$-shell emission lines. For $\omega_{K}^{r}$, values from Ref. [27] were adopted, and for self-consistency those of $\omega_{K K}$ were rescaled accordingly from the $\omega_{K K} / \omega_{K}$ ratios [2]. The precision of the method, assessed from several $K$ and $L$ x-ray line yield measurements, was found to be better than $\sim 5 \%$. For each element two reference $K \mathrm{x}$-ray transitions were used, i.e., for $\mathrm{Mg}$ the $\mathrm{Cl} K \alpha$ of $\mathrm{NaCl}$ and $\mathrm{KCl}$ samples, for $\mathrm{Al}$ the $\mathrm{K}$ $K \alpha$ and $\mathrm{Cl} K \alpha$ of $\mathrm{KCl}$, and for Si the Sc $K \alpha$ and $\mathrm{K} K \beta$. The obtained BR values for $\mathrm{Mg}$ of 1880 (373) and 1800 (357), Al of 2040(541) and 2208(605), and Si of 2625(512) and 2594(536), are indeed very close.

Results for the mean values of the branching ratios and the two-electron one-photon transitions energies are summarized in Table I and compared to theoretical predictions. Our branching ratios are also represented in Fig. 4 along with the $Z$-dependent trends of various theoretical approaches and data from heavy-ion collision experiments. Since the two-electron one-photon radiative decay corresponds to a transition between correlated multielectron initial and final atomic states, the transition rates are very sensitive to an accurate theoretical treatment of the electron inter- and intrashell interactions. Indeed, Fig. 4 evinces important differences in the calculated BR values and their $Z$ dependence alike. In contrast, the TEOP transition energies are much less sensitive, and a good agreement between our experimental values and the calculated ones is achieved. Further, the TEOP linewidths of 2.5(0.6) eV for $\mathrm{Mg}, 2.9(1.7) \mathrm{eV}$ for $\mathrm{Al}$, and 3.8(0.9) eV for $\mathrm{Si}$ were found to be $\sim 1.6$ times larger than those corresponding to the sum of the initial and final state widths (see [2]).

TABLE I. The $K \alpha^{h}$ to $K \alpha \alpha^{h}$ branching ratios and the $K \alpha \alpha^{h}$ transition energies for $\mathrm{Mg}, \mathrm{Al}$, and $\mathrm{Si}$. Also listed are different theoretical predictions.

\begin{tabular}{ccccc}
\hline \hline \multicolumn{3}{c}{ Branching ratio } & \multicolumn{2}{c}{ Energy (eV) } \\
$Z$ & Experiment & Theory & Experiment & Theory \\
\hline 12 & $1838(258)$ & $667[11]$ & $2586.7(4)$ & $2585.45[28]$ \\
& & $928[20]$ & & $2586.00[20]$ \\
& & $2417[21]^{\mathrm{a}}$ & & $2585.60[21]$ \\
13 & $2115(403)$ & $758[11]$ & $3056.5(9)$ & $3056.54[28]$ \\
& & $686[19]$ & & \\
& & $999[20]$ & & $3057.49[20]$ \\
& & $2617[21]^{\mathrm{a}}$ & & $3058.68[21]$ \\
14 & $2610(370)$ & $833[11]$ & $3568.3(4)$ & $3566[28]$ \\
& & $1126[20]$ & & $3567.43[20]$ \\
& & $3007[21]^{\mathrm{a}}$ & & $3569.37[21]$ \\
\hline \hline
\end{tabular}

${ }^{a} K \alpha^{h}$ rates in the length gauge are from Ref. [29].

${ }^{\mathrm{b}} K \alpha \alpha^{h}$ rates include the coupling of the initial and final state vacancies with the $3 p$ electron.
The present $K \alpha^{h}$ to $K \alpha \alpha^{h}$ ratios compare best to the most recent relativistic configuration interaction calculations $[21,29]$ and to the many-body perturbation theory predictions $[12,14,15]$. The branching ratios of Åberg, Jamison, and Richard [11] and Gavrila and Hansen [17] based on the relaxation of the initial and final states and the nonorthogonality of the Hartree-Fock orbitals are, however, underestimated. This is not surprising because the shake approximation is essentially a single-particle model in which only the average electron interactions (screening) are considered. In contrast, the first-order perturbation theory in the electron interaction used for calculating the correlated wave functions in the initial and final states by Kelly [12] for Fe, Amusia, Lee, and Zinoviev [15] for $\mathrm{Ne}$, Luken, Greenberg, and Vincent [16] for $\mathrm{Ni}^{18+}$, and Safranova and Senashenko [14] for He- and Li-like ions accounts for electron correlations explicitly. Predictions of Baptista [18] within the second-order perturbation theory employing screened hydrogenic basis wave functions yield, however, much lower branching ratios. Likewise, the $K \alpha \alpha^{h}$ rates are overestimated in the relativistic multiconfiguration Dirac-Fock calculations of Saha et al. [20] and also those of Costa et al. [19], who used the singleconfiguration mode. In comparison to these two sets of calculations, a more important amount of electron-electron correlations was accounted for in the relativistic configuration interaction formalism of Kadrekar and Natarajan [21]. The TEOP decay rates were calculated in the active space approximation by using multiconfiguration Dirac-Fock wave functions. The coupling between the inner-shell vacancies and the outer incomplete subshells was neglected for all elements with the exception of Al. Noteworthy is the good agreement of the calculations with

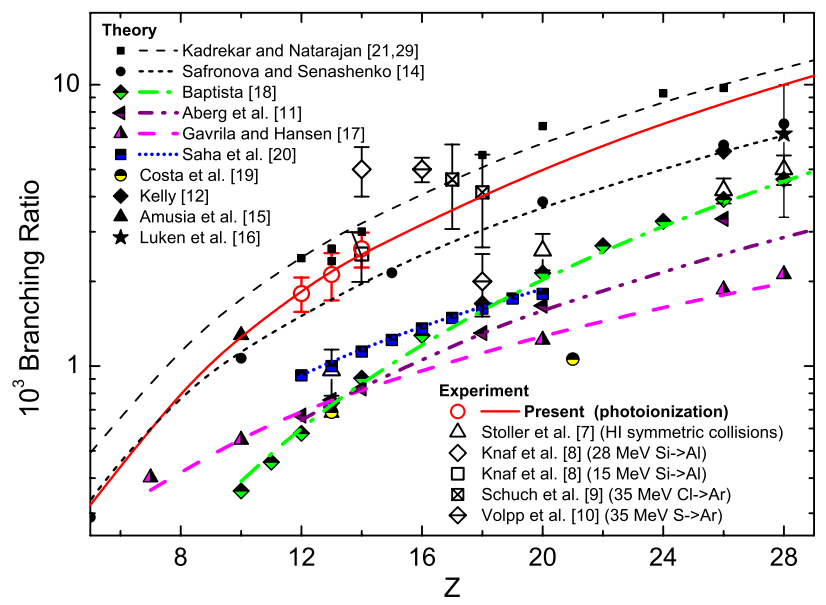

FIG. 4 (color online). Branching ratios of the one-electron to two-electron one-photon transitions for $\mathrm{Mg}, \mathrm{Al}$, and $\mathrm{Si}$ (open circles) together with theoretical predictions as a function of the atomic number $Z$. The plots through the different data sets correspond to power-law fits, whereas the solid line represents a $Z^{2}$ dependence. Experimental results from HI collision experiments are also shown for comparison. 
our Al branching ratio when the coupling effect is included, suggesting the importance of the interactions between the valence electrons and the inner-shell vacancies. It is also worthwhile to examine the $Z$-dependent trends of the branching ratios. Since an approximate $Z^{2}$ dependence for the BRs is expected, a $Z^{2}$ power-law fit to our data was applied. As shown in Fig. 4, the plot is quite close to the one for Li-like ions [14] below $Z=8$. It also coincides with the Ne TEOP decay rate of Amusia, Lee, and Zinoviev [15], where for the BR the $K \alpha^{h}$ rate from Ref. [17] was adopted. From this comparison one may tentatively conclude that owing to the presence of other $2 p$ and outer-shell electrons the BR ratios increase.

In conclusion, the first observation of the correlated twoelectron one-photon transitions in single-photon impact is reported. Unlike any of the previous experimental data, our branching ratios give an important point of comparison for the different theoretical approaches that address the manybody problem. Experiments for higher $Z$ elements and molecules, as well as with a better energy resolution, are called for. The prerequisite for such measurements and also for improving the uncertainties is a higher photon flux. Therefore, experiments with nonmonochromatized undulator radiation $\left(\sim 10^{14}\right.$ photons/s) and intense $\mathrm{x}$-ray freeelectron laser beams are envisaged.

We acknowledge the support of the Swiss National Science Foundation and the ESRF.

*joanna.hoszowska@unifr.ch

'Present address: Swiss Light Source, Paul Scherrer Institute, CH-5232 Villigen, Switzerland.

${ }^{\ddagger}$ Present address: Department of Chemistry, University of Saskatchewan, Saskatoon, Canada.

[1] J. Hoszowska et al., Phys. Rev. Lett. 102, 073006 (2009).

[2] J. Hoszowska et al., Phys. Rev. A 82, 063408 (2010).

[3] L. Young et al., Nature (London) 466, 56 (2010).
[4] W. Heisenberg, Z. Phys. 32, 841 (1925).

[5] W. Wölfli et al., Phys. Rev. Lett. 35, 656 (1975).

[6] A. R. Knudson et al., Phys. Rev. Lett. 37, 679 (1976).

[7] C. Stoller et al., Phys. Rev. A 15, 990 (1977).

[8] B. Knaf, G. Presser, and J. Stähler, Phys. Lett. 60A, 106 (1977).

[9] R. Schuch, G. Gauker, and H. Schmidt-Böcking, Z. Phys. A 290, 19 (1979).

[10] J. Volpp et al., J. Phys. B 12, L325 (1979).

[11] T. Åberg, K. A. Jamison, and P. Richard, Phys. Rev. Lett. 37, 63 (1976).

[12] H. P. Kelly, Phys. Rev. Lett. 37, 386 (1976).

[13] C. Stoller et al., Phys. Lett. 58A, 18 (1976).

[14] U. I. Safranova and V. S. Senashenko, J. Phys. B 10, L271 (1977).

[15] M. Ya. Amusia, L. S. Lee, and A. N. Zinoviev, Phys. Lett. 60A, 300 (1977).

[16] W. L. Luken, J. S. Greenberg, and P. Vincent, Phys. Rev. A 15, 2305 (1977).

[17] M. Gavrila and J. E. Hansen, J. Phys. B 11, 1353 (1978).

[18] G. B. Baptista, J. Phys. B 17, 2177 (1984).

[19] A. M. Costa et al., J. Phys. B 39, 2355 (2006).

[20] J. K. Saha et al., Phys. Lett. A 373, 252 (2009).

[21] R. Kadrekar and L. Natarajan, J. Phys. B 43, 155001 (2010).

[22] R. Diamant et al., Phys. Rev. A 62, 052519 (2000).

[23] J. Szlachetko et al., J. Synchrotron Radiat. 17, 400 (2010).

[24] R. D. Deslattes et al., Rev. Mod. Phys. 75, 35 (2003).

[25] J. Hoszowska et al., Nucl. Instrum. Methods Phys. Res., Sect. A 376, 129 (1996).

[26] http://physics.nist.gov/xcom.

[27] W. Bambynek, in Proceedings of the X84 International Conference on X-ray and Inner Shell Processes in Atoms, Molecules and Solids, Leipzig, 1984, edited by A. Meisel and J. Finster (VEB Press, Langensalza, 1984).

[28] M. C. Martins et al., J. Phys. B 37, 3785 (2004).

[29] L. Natarajan, Phys. Rev. A 78, 052505 (2008).

[30] T. Åberg and J. Utriainen, Phys. Rev. Lett. 22, 1346 (1969). 